\title{
How to Compute the Information Content of 3-FR, 3-DP Decoupled Designs with Uniform Probability Density Functions for Their FRs
}

\author{
João Fradinho ${ }^{1, *}$, Miguel Cavique ${ }^{2}$, António Gabriel-Santos ${ }^{1}$, António Mourão $^{1}$ and António Gonçalves-Coelho ${ }^{1}$ \\ ${ }^{1}$ UNIDEMI \& DEMI, Faculdade de Ciências e Tecnologia, Universidade Nova de Lisboa, Campus de Caparica, 2829-516 Caparica, \\ Portugal \\ ${ }^{2}$ UNIDEMI, CINAV \& Escola Naval, Base Naval de Lisboa - Alfeite, 2810-001 Almada, Portugal
}

\begin{abstract}
The computation of the information content of decoupled designs implies the use of conditional probability, because the existing design parameters must be adjusted in a certain order, as to fulfil all the existing functional requirements at the first trial. Nevertheless, the computation of the information content is hard to perform when the system range of the functional requirements is denoted by probability density functions. In their works, Suh and Park proposed a graphical method to compute the information content of 2-FR, 2-DP decoupled designs when the probability density functions of the FRs are uniform. This paper introduces an extension to the Suh and Park's previous findings. It presents a method that uses analytical and descriptive geometry techniques to compute the information content of 3-FR, 3-DP decoupled designs with uniform probability density distributions of their FRs, and an illustrative example is presented as well.
\end{abstract}

\section{Introduction}

Most of the current engineering design methodologies follow the costly and time-consuming trial-and-error pattern, as for example the ones proposed by Pahl \& Beitz [1] and by Hubka [2]. Yet, cost and speed of the engineering design processes became critical by the end of the last century due to the increasing demand of better, faster, cheaper or more capable new products with a short time-to-market prerequisite.

In the late 1970s, N. P. Suh et al. [3] introduced a new design theory that was made widely known under the name of Axiomatic Design (AD) [4]. Suh's motivation was to provide scholars and engineers with a sound theoretical foundation for design that conforms to the criteria of modern science, as to foster significant advances in teaching and in practicing design. As a result, $\mathrm{AD}$ can be used to teach engineering design in a manner that is similar to mathematics or physics, and allows engineers to consistently avoid the trial-and-error design development cycle, which lets it easier to do it right at the first time, timely and at an affordable cost.

In $\mathrm{AD}$, design is seen as a decision-making process, which success depends on the formal awareness on the functional objectives and constraints, as well as on understanding the engineering sciences and the technologies associated to the likely alternative design solutions.

The design decisions correspond to the mapping between each pair of adjacent design domains, namely the Customer Domain, the Functional Domain, the Physical Domain, and the Process Domain, as depicted in Figure 1.

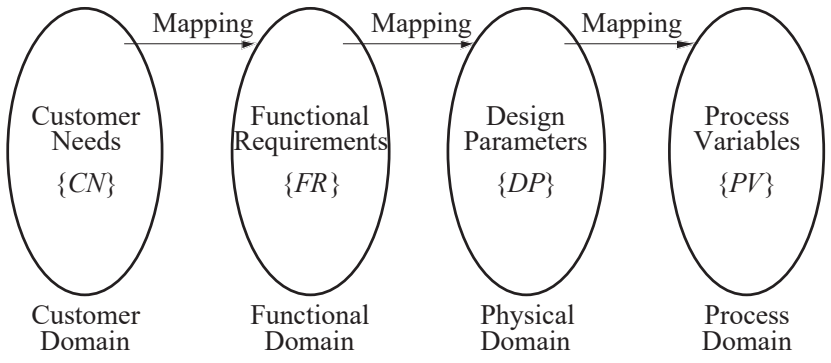

Fig. 1. The design domains.

Each design solution is represented by a design equation. For example, the mapping between the functional and the physical domains is of the form

$$
\{F R\}=[A]\{D P\}
$$

where $\{F R\}$ is the vector of the functional requirements, $\{D P\}$ is the vector of the design parameters, and $[A]$ is the design matrix, for which

$$
A_{i j}=\frac{\partial F R_{i}}{\partial D P_{j}}, i=1, . . m, j=1, . . n
$$

The AD fundamental decision criteria are based on the independence axiom and on the minimum information axiom [4].

For the cases where $m=n$, two kinds of design solutions fulfil the independence axiom: the uncoupled designs, in which the values of the design parameters

\footnotetext{
* Corresponding author: jmf@fct.unl.pt
} 
(DPs) can be adjusted in an arbitrary order as to attain the prescribed values for the FRs; and the decoupled designs, in which the values of the DPs can be set in a certain order, so that setting the value of each DP only impacts one FR. Therefore, the uncoupled designs have diagonal design matrices, while decoupled designs have triangular design matrices. A third kind of designs with $m=n$ - the coupled designs - does not conform to the independence axiom and should be avoided [4].

According to AD's theorem 1, designs with $m>n$ are coupled, while designs with $m<n$ are redundant. Redundant designs can be reduced to uncoupled designs or to decoupled designs, or even to coupled designs, as per AD's theorem 3 [5], depending on the manner their design matrices are populated [6].

The Minimum Information Axiom is used to compare multiple design solutions i.e., when multiple sets of different DPs can be used to fulfil the same set of FRs in an independent manner.

The concept of information content is the same that was adopted in the Shannon's theory of communication [7].

The computation of the information content of a 1FR, 1-DP design can be explained as follows.

Figure 2 depicts the probability density function (pdf) of fulfilling the system's FR. In the same figure, the system range represents the whole ability range of the system, while the design range is the working range that the designer is looking for [4]. The greyed area represents the so-called common range of the design.

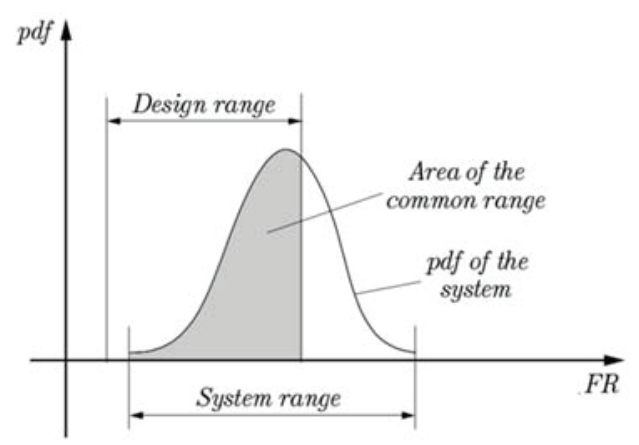

Fig. 2. The system range, the design range and the common range of a 1-FR design.

The probability density function relates to the probability of success of the system, so that the whole area of the system range is such that

$$
\int_{-\infty}^{+\infty} p d f(F R) d F R=1
$$

The probability of the design fulfilling the FR within the design range is given by

$$
p=\frac{\text { Area of the common range }}{\text { Area of the system range }}
$$

The information content of the design, $I$, expressed in bit, is computed trough

$$
I=\log _{2} \frac{1}{p}=-\log _{2} p
$$

For the case of an $m$-FR design, the total probability of success, $p_{T}$, is

$$
p_{T}=\prod_{i=1}^{m} p_{i}
$$

where $p_{i}$ is the probability of success of $F R_{i}$.

Thus, the information content of the design is

$$
I_{T}=\sum_{i=1}^{m} I_{i}
$$

Suh [8, pp 179-185] and Park [9, pp 38-51] showed that a graphical method could be used to compute the information content of 2-FR, 2-DP decoupled designs when the probability density functions of both FRs have a uniform distribution. The present work contains an extension to the aforesaid method applied to 3-FR, 3-DP decoupled designs with uniform probability density functions of their FRs.

\section{The information content of 3-FR, 3-DP decoupled designs}

The computation of the information content of multipleFR designs is simple for uncoupled designs because the probability of fulfilling each FR does not depend on the remaining FRs. However, this is not the case of the decoupled designs, for which the probability is conditional.

According to Equation (1), the design equation of a 2-FR, 2-DP decoupled design is

$$
\left\{\begin{array}{l}
F R_{1} \\
F R_{2}
\end{array}\right\}=\left[\begin{array}{ll}
A_{11} & 0 \\
A_{21} & A_{22}
\end{array}\right]\left\{\begin{array}{c}
D P_{1} \\
D P_{2}
\end{array}\right\}
$$

Hence, the random variations of the FRs due to the random variations of the DPs are given by

$$
\left\{\begin{array}{c}
\delta F R_{1} \\
\delta F R_{2}
\end{array}\right\}=\left[\begin{array}{cc}
A_{11} & 0 \\
A_{21} & A_{22}
\end{array}\right]\left\{\begin{array}{c}
\delta D P_{1} \\
\delta D P_{2}
\end{array}\right\}
$$

Equation (9) shows that $\delta F R_{1}$ is statistically independent, while $\delta F R_{2}$ is statistically dependent with respect to $\delta F R_{1}$.

In short, Park [9, p. 40] showed that the problem stated by Equation (9) can be depicted in the physical domain by Figure 3, for the case of $A_{i j} \geq 0$ and

$$
\begin{aligned}
& -\Delta F R_{i} \leq \delta F R_{i} \leq \Delta F R_{i}, \Delta F R_{i} \geq 0 \\
& -\Delta D P_{i} \leq \delta D P_{i} \leq \Delta D P_{i}, \Delta D P_{i} \geq 0
\end{aligned}
$$




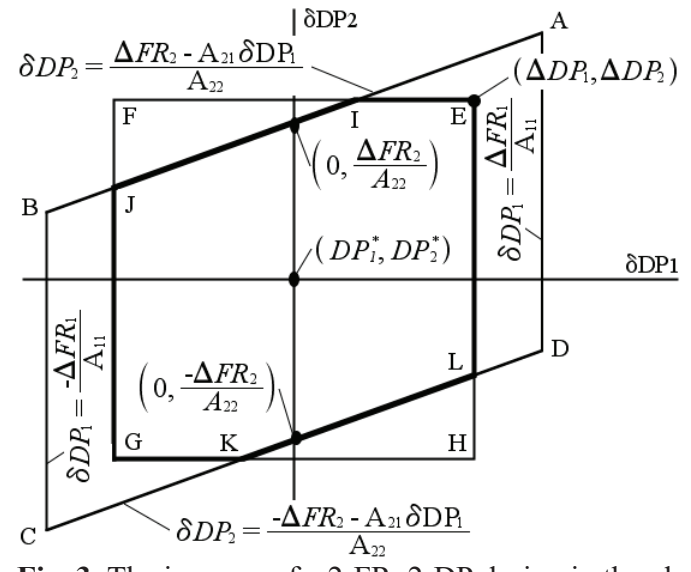

Fig. 3. The isogram of a 2-FR, 2-DP design in the physical domain [9].

The parallelogram ABCD of Figure 3 is the system range of the design, while the quadrilateral EFGH is the design range and the hexagon IJGKLE is the common range. Thus, for the case of uniform probability density functions, the information content is given by

$$
I_{u}=\log _{2} \frac{1}{p}=\log _{2}\left(\frac{\text { Area of quadrilateral EFGH }}{\text { Area of hexagon IJGKLE }}\right)
$$

The areas of the quadrilateral EFGH and of the hexagon IJGKLE can be computed by integration, using the equations that were found by Park [9] and that are included in Figure 3. Yet, those areas can be much more easily evaluated using a 2-D CAD system capable of measuring areas of closed polygons.

This method can be extended to 3-FR, 3-DP decoupled designs with uniform pdfs. The random variation of the functional requirement, $\delta F R_{i}$, due to the random variation in a design parameter, $\delta D P_{j}$, is expressed as

$$
\left[\begin{array}{c}
\delta F R_{1} \\
\delta F R_{2} \\
\delta F R_{3}
\end{array}\right]=\left[\begin{array}{ccc}
\frac{\partial F R_{1}}{\partial D P_{1}} & 0 & 0 \\
\frac{\partial F R_{2}}{\partial D P_{1}} & \frac{\partial F R_{2}}{\partial D P_{2}} & 0 \\
\frac{\partial F R_{3}}{\partial D P_{1}} & \frac{\partial F R_{3}}{\partial D P_{2}} & \frac{\partial F R_{3}}{\partial D P_{3}}
\end{array}\right]\left[\begin{array}{c}
\delta D P_{1} \\
\delta D P_{2} \\
\delta D P_{3}
\end{array}\right]
$$
we have

Denoting the elements of the design matrix by $A_{i j}$,

$$
\left[\begin{array}{l}
\delta F R_{1} \\
\delta F R_{2} \\
\delta F R_{3}
\end{array}\right]=\left[\begin{array}{ccc}
A_{11} & 0 & 0 \\
A_{21} & A_{22} & 0 \\
A_{31} & A_{32} & A_{33}
\end{array}\right]\left[\begin{array}{c}
\delta D P_{1} \\
\delta D P_{2} \\
\delta D P_{3}
\end{array}\right]
$$

Now, let us suppose the following unbiased tolerance ranges:

$$
\begin{aligned}
& -\Delta F R_{i} \leq \delta F R_{i} \leq \Delta F R_{i} \\
& -\Delta D P_{j} \leq \delta D P_{j} \leq \Delta D P_{j}
\end{aligned}
$$

Let us also suppose that

$$
\Delta F R_{i} \geq 0 ; \Delta D P_{j} \geq 0 ; A_{i j} \geq 0
$$

and that

$\delta D P_{j}$ are statistically independent;

$\delta F R_{2}$ is statistically dependent to $\delta F R_{1}$;

$\delta F R_{3}$ is statistically dependent to $\delta F R_{1}$ and $\delta F R_{2}$.

Under these conditions, we have

$$
\begin{gathered}
\delta F R_{1}=A_{11} \delta D P_{1} \\
\delta F R_{2}=A_{21} \delta D P_{1}+A_{22} \delta D P_{2} \\
\delta F R_{3}=A_{31} \delta D P_{1}+A_{32} \delta D P_{2}+A_{33} \delta D P_{3}
\end{gathered}
$$

so that

$$
\begin{gathered}
\delta D P_{1}=\frac{\delta F R_{1}}{A_{11}} \\
\delta D P_{2}=\frac{\delta F R_{2}}{A_{22}}-\frac{A_{21}}{A_{22}} \delta D P_{1} \\
\delta D P_{3}=\frac{\delta F R_{3}}{A_{33}}-\frac{A_{32}}{A_{33}} \delta D P_{2}-\frac{A_{31}}{A_{33}} \delta D P_{1}
\end{gathered}
$$

The combination of Equations (15), (16) and (18) gives

$$
\begin{aligned}
& -\Delta F R_{1} \leq A_{11} \delta D P_{1} \leq \Delta F R_{1} \\
& -\Delta F R_{2} \leq A_{21} \delta D P_{1}+A_{22} \delta D P_{2} \leq \Delta F R_{2} \\
& -\Delta F R_{3} \leq A_{31} \delta D P_{1}+A_{32} \delta D P_{2}+A_{33} \delta D P_{3} \leq \Delta F R_{3} \\
& -\Delta D P_{1} \leq \delta D P_{1} \leq \Delta D P_{1} \\
& -\Delta D P_{2} \leq \delta D P_{2} \leq \Delta D P_{2} \\
& -\Delta D P_{3} \leq \delta D P_{3} \leq \Delta D P_{3}
\end{aligned}
$$

Equation (19) represents the limits of the design in the physical domain. The first three conditions characterize the system range and the last three denote the design range.

The system range is the intersection of three pairs of semi-spaces. The first condition represents two profile planes that are parallel to the projection plane $\left(\delta D P_{2} \mathrm{O} \delta D P_{3}\right)$; the second one represents two vertical planes that are parallel to $\delta \mathrm{DP}_{3}$ axis; and the third condition represents two planes that are oblique to the three projection planes.

Figure 4 shows the intersections of the six planes with the orthogonal projection planes. The values of $A_{i j}$, $\triangle F R_{i}$ and $\triangle D P_{j}$ were considered as positive in the graphic representation. Thus, the vertical and the oblique planes open to the right.

The design range is a quadrangular prism that is also centred in the origin.

The bold line solid of Figure 5 is the 10-face polyhedron [AA','II'JJ'KL'C',C'MM'NN'PQ'] that represents the common range of the problem under study. It is obtained through the intersection of the solids that represent the system and the design ranges. 


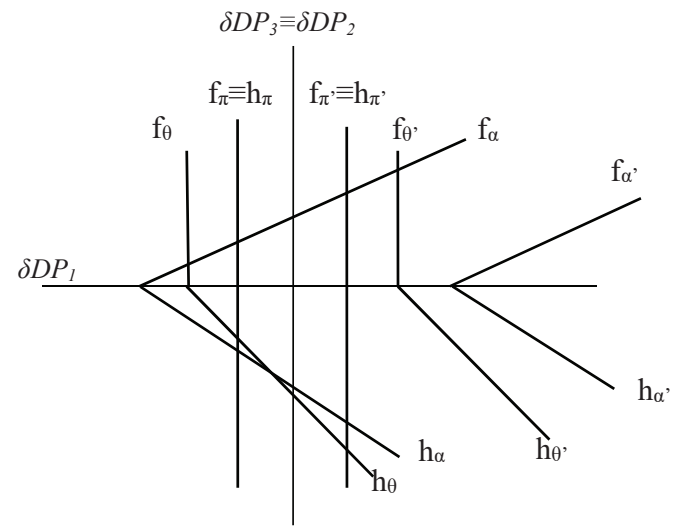

Fig. 4. The six planes that determine the system range in the DP domain.

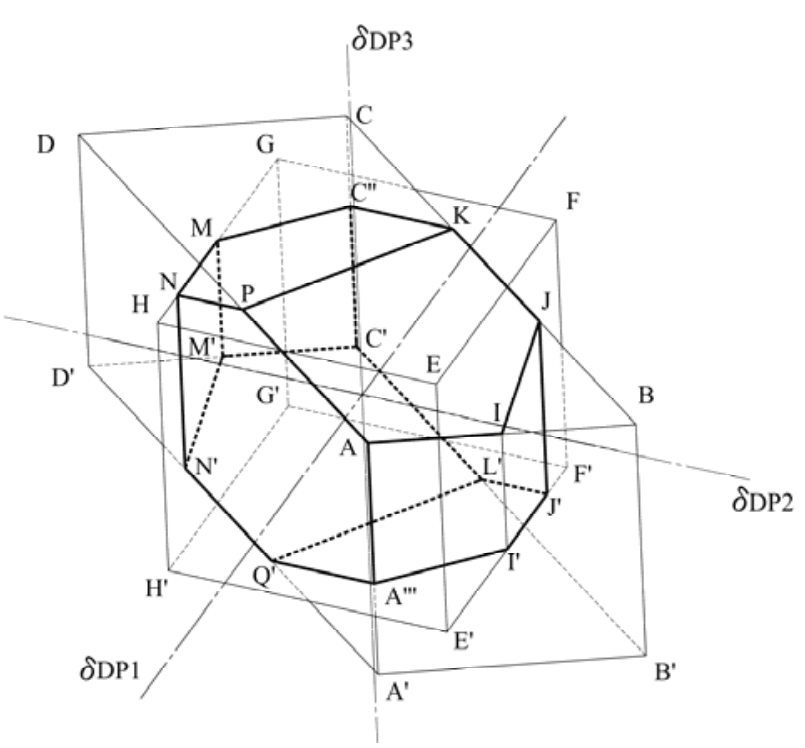

Fig. 5. The system, the design and the common ranges.

The system range is the 6-face polyhedron [ABCDA'B'C'D'] with two profile faces ([ADA'D'] and [BCB' $\left.\left.\mathrm{C}^{\prime}\right]\right)$, which belong to planes $\pi$ and $\pi$ '; two vertical faces ([ABA'B'] and [CDC'D']), belonging to planes $\theta$ and $\theta^{\prime}$; and two oblique faces ([ABCD] and [A'B'C'D']) that belong to planes $\alpha$ and $\alpha$ '.

The design range is the quadrangular prism [EFGHE'F'G'H'] with faces parallel to the projection planes.

The coordinates of the vertices of the system and of the design ranges of Figure 5 are:

$$
A\left(\begin{array}{l}
\frac{\Delta F R_{1}}{A_{11}} ; \\
\frac{\Delta F R_{2}}{A_{22}}-\frac{A_{21}}{A_{22}} \frac{\Delta F R_{1}}{A_{11}} ; \\
\frac{\Delta F R_{3}}{A_{33}}-\frac{A_{32}}{A_{33}} \frac{\Delta F R_{2}}{A_{22}}-\left(\frac{A_{31}}{A_{33}}-\frac{A_{32}}{A_{33}} \frac{A_{21}}{A_{22}}\right) \frac{\Delta F R_{1}}{A_{11}}
\end{array}\right)
$$

$$
\begin{aligned}
& A^{\prime}\left(\begin{array}{l}
\frac{\Delta F R_{1}}{A_{11}} ; \\
\frac{\Delta F R_{2}}{A_{22}}-\frac{A_{21}}{A_{22}} \frac{\Delta F R_{1}}{A_{11}} ; \\
-\frac{\Delta F R_{3}}{A_{33}}-\frac{A_{32}}{A_{33}} \frac{\Delta F R_{2}}{A_{22}}-\left(\frac{A_{31}}{A_{33}}-\frac{A_{32}}{A_{33}} \frac{A_{21}}{A_{22}}\right) \frac{\Delta F R_{1}}{A_{11}}
\end{array}\right) \\
& B\left(\begin{array}{l}
-\frac{\Delta F R_{1}}{A_{11}} ; \\
\frac{\Delta F R_{2}}{A_{22}}+\frac{A_{21}}{A_{22}} \frac{\Delta F R_{1}}{A_{11}} ;
\end{array}\right. \\
& \left(\frac{\Delta F R_{3}}{A_{33}}-\frac{A_{32}}{A_{33}} \frac{\Delta F R_{2}}{A_{22}}-\left(-\frac{A_{31}}{A_{33}}+\frac{A_{32}}{A_{33}} \frac{A_{21}}{A_{22}}\right) \frac{\Delta F R_{1}}{A_{11}}\right) \\
& B^{\prime}\left(\begin{array}{l}
-\frac{\Delta F R_{1}}{A_{11}} ; \\
\frac{\Delta F R_{2}}{A_{22}}+\frac{A_{21}}{A_{22}} \frac{\Delta F R_{1}}{A_{11}} ; \\
-\frac{\Delta F R_{3}}{A_{33}}-\frac{A_{32}}{A_{33}} \frac{\Delta F R_{2}}{A_{22}}-\left(-\frac{A_{31}}{A_{33}}+\frac{A_{32}}{A_{33}} \frac{A_{21}}{A_{22}}\right) \frac{\Delta F R_{1}}{A_{11}}
\end{array}\right) \\
& -\frac{\Delta F R_{1}}{A_{11}} \\
& C-\frac{\Delta F R_{2}}{A_{22}}+\frac{A_{21}}{A_{22}} \frac{\Delta F R_{1}}{A_{11}} \text {; } \\
& \left.\frac{\Delta F R_{3}}{A_{33}}+\frac{A_{32}}{A_{33}} \frac{\Delta F R_{2}}{A_{22}}-\left(-\frac{A_{31}}{A_{33}}+\frac{A_{32}}{A_{33}} \frac{A_{21}}{A_{22}}\right) \frac{\Delta F R_{1}}{A_{11}}\right) \\
& C^{\prime}\left(\begin{array}{l}
-\frac{\Delta F R_{1}}{A_{11}} ; \\
-\frac{\Delta F R_{2}}{A_{22}}+\frac{A_{21}}{A_{22}} \frac{\Delta F R_{1}}{A_{11}} ; \\
-\frac{\Delta F R_{3}}{A_{33}}+\frac{A_{32}}{A_{33}} \frac{\Delta F R_{2}}{A_{22}}-\left(-\frac{A_{31}}{A_{33}}+\frac{A_{32}}{A_{33}} \frac{A_{21}}{A_{22}}\right) \frac{\Delta F R_{1}}{A_{11}}
\end{array}\right) \\
& D\left(\begin{array}{l}
\frac{\Delta F R_{1}}{A_{11}} ; \\
-\frac{\Delta F R_{2}}{A_{22}}-\frac{A_{21}}{A_{22}} \frac{\Delta F R_{1}}{A_{11}} ; \\
\frac{\Delta F R_{3}}{A_{33}}+\frac{A_{32}}{A_{33}} \frac{\Delta F R_{2}}{A_{22}}-\left(\frac{A_{31}}{A_{33}}-\frac{A_{32}}{A_{33}} \frac{A_{21}}{A_{22}}\right) \frac{\Delta F R_{1}}{A_{11}}
\end{array}\right) \\
& D^{\prime}\left(\begin{array}{l}
\frac{\Delta F R_{1}}{A_{11}} ; \\
-\frac{\Delta F R_{2}}{A_{22}}-\frac{A_{21}}{A_{22}} \frac{\Delta F R_{1}}{A_{11}} ; \\
-\frac{\Delta F R_{3}}{A_{33}}+\frac{A_{32}}{A_{33}} \frac{\Delta F R_{2}}{A_{22}}-\left(\frac{A_{31}}{A_{33}}-\frac{A_{32}}{A_{33}} \frac{A_{21}}{A_{22}}\right) \frac{\Delta F R_{1}}{A_{11}}
\end{array}\right)
\end{aligned}
$$




$$
\begin{gathered}
\mathrm{E}\left(\Delta D P_{1} ; \Delta D P_{2} ; \Delta D P_{3}\right) \\
\mathrm{E}^{\prime}\left(\Delta D P_{1} ; \Delta D P_{2} ;-\Delta D P_{3}\right) \\
\mathrm{F}\left(-\Delta D P_{1} ; \Delta D P_{2} ; \Delta D P_{3}\right) \\
\mathrm{F}^{\prime}\left(-\Delta D P_{1} ; \Delta D P_{2} ;-\Delta D P_{3}\right) \\
\mathrm{G}\left(-\Delta D P_{1} ;-\Delta D P_{2} ; \Delta D P_{3}\right) \\
\mathrm{G}^{\prime}\left(-\Delta D P_{1} ;-\Delta D P_{2} ;-\Delta D P_{3}\right) \\
\mathrm{H}\left(\Delta D P_{1} ;-\Delta D P_{2} ; \Delta D P_{3}\right) \\
\mathrm{H}^{\prime}\left(\Delta D P_{1} ;-\Delta D P_{2} ;-\Delta D P_{3}\right)
\end{gathered}
$$

The coordinates of the vertices of the common range depend on the six planes $\left(\pi, \pi^{\prime}, \theta, \theta^{\prime}, \alpha, \alpha^{\prime}\right)$ that define the system range, as well as on the six planes parallel to the axes that define the design range, herewith named by their own coordinates:

$$
\left(\Delta D P_{1}, \Delta D P_{2}, \Delta D P_{3},-\Delta D P_{1},-\Delta D P_{2},-\Delta D P_{3}\right) \text {. }
$$

Each vertex is the intersection of three of these 12 planes, as follows

$$
\begin{gathered}
\mathrm{A}(\pi \cap \theta \cap \alpha) \\
\mathrm{A}^{\prime \prime}\left(\pi \cap \theta \cap-\Delta D P_{3}\right) \\
\mathrm{I}\left(\theta \cap \Delta D P_{2} \cap \alpha\right) \\
\mathrm{I}^{\prime}\left(\theta \cap \Delta D P_{2} \cap-\Delta D P_{3}\right) \\
\mathrm{J}\left(\pi^{\prime} \cap \Delta D P_{2} \cap \alpha\right) \\
\mathrm{J}^{\prime}\left(\pi^{\prime} \cap \Delta D P_{2} \cap-\Delta D P_{3}\right) \\
\mathrm{K}\left(\pi^{\prime} \cap \alpha \cap \Delta D P_{3}\right) \\
\mathrm{L}^{\prime}\left(\pi^{\prime} \cap \alpha^{\prime} \cap-\Delta D P_{3}\right) \\
\mathrm{C}^{\prime \prime}\left(\pi^{\prime} \cap \theta^{\prime} \cap \Delta D P_{3}\right) \\
\mathrm{C}^{\prime}\left(\pi^{\prime} \cap \theta^{\prime} \cap \alpha^{\prime}\right) \\
\mathrm{M}\left(\theta^{\prime} \cap-\Delta D P_{2} \cap \Delta D P_{3}\right) \\
\mathrm{M}^{\prime}\left(\theta ' \cap-\Delta D P_{2} \cap \alpha^{\prime}\right) \\
\mathrm{N}\left(\pi \cap-\Delta D P_{2} \cap \Delta D P_{3}\right) \\
\mathrm{N}^{\prime}\left(\pi \cap-\Delta D P_{2} \cap \alpha^{\prime}\right) \\
\mathrm{P}\left(\pi \cap \alpha \cap \Delta D P_{3}\right) \\
\mathrm{Q}^{\prime}\left(\pi \cap \alpha^{\prime} \cap-\Delta D P_{3}\right)
\end{gathered}
$$

The common range is a 10-face, 16-vertex, 22-edge polyhedron, which confirms the Euler's formula.
The coordinates of the vertices are:

$$
\begin{aligned}
& \frac{\Delta F R_{1}}{A_{11}} \\
& \mathrm{~A} \frac{\Delta F R_{2}}{A_{22}}-\frac{A_{21}}{A_{22}} \frac{\Delta F R_{1}}{A_{11}} \text {; } \\
& \left.\frac{\Delta F R_{3}}{A_{33}}-\frac{A_{32}}{A_{33}} \frac{\Delta F R_{2}}{A_{22}}-\left(\frac{A_{31}}{A_{33}}-\frac{A_{32}}{A_{33}} \frac{A_{21}}{A_{22}}\right) \frac{\Delta F R_{1}}{A_{11}}\right) \\
& \mathrm{A}^{\prime \prime \prime}\left(\frac{\Delta F R_{1}}{A_{11}} ; \frac{\Delta F R_{2}}{A_{22}}-\frac{A_{21}}{A_{22}} \frac{\Delta F R_{1}}{A_{11}} ;-\Delta D P_{3}\right) \\
& \frac{\Delta F R_{2}}{A_{21}}-\frac{A_{22}}{A_{21}} \Delta D P_{2} \\
& \text { I } \triangle D P_{2} \text {; } \\
& \left(\frac{\Delta F R_{3}}{A_{33}}-\frac{A_{31}}{A_{33}} \frac{\Delta F R_{2}}{A_{21}}+\left(\frac{A_{31}}{A_{33}} \frac{A_{22}}{A_{21}}-\frac{A_{32}}{A_{33}}\right) \Delta D P_{2}\right) \\
& \mathrm{I}^{\prime}\left(\frac{\Delta F R_{2}}{A_{21}}-\frac{A_{22}}{A_{21}} \Delta D P_{2} ; \Delta D P_{2} ;-\Delta D P_{3}\right) \\
& \mathrm{J}\left(-\frac{\Delta F R_{1}}{A_{11}} ; \Delta D P_{2} ; \frac{\Delta F R_{3}}{A_{33}}+\frac{A_{31}}{A_{33}} \frac{\Delta F R_{1}}{A_{11}}-\frac{A_{32}}{A_{33}} \Delta D P_{2}\right) \\
& \mathrm{J}^{\prime}\left(-\frac{\Delta F R_{1}}{A_{11}} ; \Delta D P_{2} ;-\triangle D P_{3}\right) \\
& \mathrm{K}\left(-\frac{\Delta F R_{1}}{A_{11}} ; \frac{\Delta F R_{3}}{A_{32}}+\frac{A_{31}}{A_{32}} \frac{\Delta F R_{1}}{A_{11}}-\frac{A_{33}}{A_{32}} \Delta D P_{3} ; \Delta D P_{3}\right) \\
& \mathrm{L}^{\prime}\left(-\frac{\Delta F R_{1}}{A_{11}} ;-\frac{\Delta F R_{3}}{A_{32}}+\frac{A_{31}}{A_{32}} \frac{\Delta F R_{1}}{A_{11}}+\frac{A_{33}}{A_{32}} \Delta D P_{3} ;-\Delta D P_{3}\right) \\
& \mathrm{C}^{\prime \prime}\left(-\frac{\Delta F R_{1}}{A_{11}} ;-\frac{\Delta F R_{2}}{A_{22}}+\frac{A_{21}}{A_{22}} \frac{\Delta F R_{1}}{A_{11}} ; \Delta D P_{3}\right) \\
& \mathrm{C}^{\prime}\left(\begin{array}{l}
-\frac{\Delta F R_{1}}{A_{11}} ; \\
-\frac{\Delta F R_{2}}{A_{22}}+\frac{A_{21}}{A_{22}} \frac{\Delta F R_{1}}{A_{11}} ; \\
-\frac{\Delta F R_{3}}{A_{33}}+\frac{A_{32}}{A_{33}} \frac{\Delta F R_{2}}{A_{22}}-\left(-\frac{A_{31}}{A_{33}}+\frac{A_{32}}{A_{33}} \frac{A_{21}}{A_{22}}\right) \frac{\Delta F R_{1}}{A_{11}}
\end{array}\right) \\
& \mathrm{M}\left(-\frac{\Delta F R_{2}}{A_{21}}+\frac{A_{22}}{A_{21}} \Delta D P_{2} ;-\triangle D P_{2} ; \Delta D P_{3}\right) \\
& \mathrm{M}^{\prime}\left(\begin{array}{l}
-\frac{\Delta F R_{2}}{A_{21}}+\frac{A_{22}}{A_{21}} \Delta D P_{2} ; \\
-\Delta D P_{2} ; \\
-\frac{\Delta F R_{3}}{A_{33}}+\frac{A_{31}}{A_{33}} \frac{\Delta F R_{2}}{A_{21}}-\left(\frac{A_{31}}{A_{33}} \frac{A_{22}}{A_{21}}-\frac{A_{32}}{A_{33}}\right) \Delta D P_{2}
\end{array}\right) \\
& \mathrm{N}\left(\frac{\Delta F R_{1}}{A_{11}} ;-\Delta D P_{2} ; \Delta D P_{3}\right)
\end{aligned}
$$




$$
\begin{gathered}
\mathrm{N}^{\prime}\left(\frac{\Delta F R_{1}}{A_{11}} ;-\Delta D P_{2} ;-\frac{\Delta F R_{3}}{A_{33}}-\frac{A_{31}}{A_{33}} \frac{\Delta F R_{1}}{A_{11}}+\frac{A_{32}}{A_{33}} \Delta D P_{2}\right) \\
\mathrm{P}\left(\frac{\Delta F R_{1}}{A_{11}} ; \frac{\Delta F R_{3}}{A_{32}}-\frac{A_{31}}{A_{32}} \frac{\Delta F R_{1}}{A_{11}}-\frac{A_{33}}{A_{32}} \Delta D P_{3} ; \Delta D P_{3}\right) \\
\mathrm{Q}^{\prime}\left(\frac{\Delta F R_{1}}{A_{11}} ;-\frac{\Delta F R_{3}}{A_{32}}-\frac{A_{31}}{A_{32}} \frac{\Delta F R_{1}}{A_{11}}+\frac{A_{33}}{A_{32}} \Delta D P_{3} ;-\Delta D P_{3}\right)
\end{gathered}
$$

\section{An illustrative example}

The probability of success of a design, $p_{s}$, as well as of its information content, $I$, are given by

$$
\begin{aligned}
& p_{s}=\frac{V_{c}}{V_{d p}} \\
& I=-\log _{2} p_{s}
\end{aligned}
$$

where $V_{c}$ is the volume of the common range and $V_{d p}$ is the tolerance volume for the design parameters, which is the design range.

The design depicted in Figure 5 will be used as an illustrative example, where it was assumed that

$$
\begin{gathered}
\Delta F R_{1}=2 \text { length units } \\
\Delta F R_{2}=3 \text { length units } \\
\Delta F R_{3}=4 \tan 35^{\circ} \text { length units } \\
A_{11}=A_{22}=A_{33}=1 \\
\Delta D P_{1}=\Delta D P_{2}=\Delta D P_{3}=3 \text { length units }
\end{gathered}
$$

As a result, the design equation of this decoupled deign is

$$
\left[\begin{array}{l}
F R_{1} \\
F R_{2} \\
F R_{3}
\end{array}\right]=\left[\begin{array}{ccc}
1 & 0 & 0 \\
1 & 1 & 0 \\
\tan 35^{\circ} & 1 & 1
\end{array}\right]\left[\begin{array}{c}
D P_{1} \\
D P_{2} \\
D P_{3}
\end{array}\right]
$$

This means that the intersection of the profile planes ( $\pi$ and $\pi^{\prime}$ ) crosses the $d D P_{1}$ axis at points 2 and -2 , the vertical planes $\left(\theta\right.$ and $\left.\theta^{\prime}\right)$ at 3 and -3 , and the oblique planes $\left(\alpha\right.$ and $\left.\alpha^{\prime}\right)$ at 4 and -4 . In addition, the vertical planes open $45^{\circ}$ to the right and the oblique planes open $35^{\circ}$ to the right, horizontally and frontally.

Thus, the volume of the common range, $V_{c}$, is the volume of the polyhedron [AA','II'JJ'KL'C',C'MM'NN'PQ']. This volume can be computed, although very hard to attain.

Therefore, a 3D solid modeller software was used to evaluate $V_{c}$.

$$
V_{c}=90,31 \text { volume units }
$$

The volume of the tolerance region for the design parameters, $V_{d p}$, is

$V_{d p}=8 \times \Delta D P_{1} \times \Delta D P_{2} \times \Delta D P_{3}=216,00$ vol. units

Thus, the probability of success and the information content are given by

$$
\begin{gathered}
p_{s}=\frac{90,31}{216,00}=0,42 \\
I=-\log _{2} p_{s}=1,26 \mathrm{bit}
\end{gathered}
$$

\section{Discussion}

As one can see, Figure 5 is the extension for the case of a design with 3-FR, 3-DP of the 2-FR, 2-DP problem that is depicted in Figure 3.

Section 4 of this paper shows how difficult can be the computation of the information content of a 3-FR, 3-DP design, even for designs with uniform pdfs, as is the case of Figure 5. In the same section, one can also see how easy can be the computation of the information content using an advanced 3D solid modeller.

It would be easy to prove that if $\triangle D P_{1}, \triangle D P_{2}$ and $\triangle D P_{3}$ were sufficiently small, then the whole design range would be contained in the system range and the information content would be null.

\section{Conclusions}

The computation of the information content of uncoupled designs is easy, independently of the number of FRs, because the involved probabilities are always independent. However, the most arduous issue of Axiomatic Design is the computation of the information content in all the other cases.

In the case of 2-FR, 2-DP decoupled designs with uniform pdfs, where conditional probability is present, Suh and Park showed that the computation is relatively easy through numerical or graphical computation.

The present paper deals with 3-FR, 3-DP decoupled designs with uniform pdfs. This is a much more difficult problem to solve than the solution presented by the two aforesaid authors. In fact, Figure 3 shows that the common range of the 2-FR, 2-DP designs is a 6-vertex polygon, while the common range of a 3-FR, 3-DP design is a 16-vertix polyhedron, as is can be ascertained through Figure 5.

Given that it is still possible to represent the last problem in a $3 \mathrm{D}$ space, than one can overtake the hard to implement the numerical computation of the information content through the use of an advanced solid modeller, as it was done in Figure 5.

Although it seems a very small advance in the stateof-art, the possibility to easily solve 3-FR, 3-DP designs with uniform pdfs by using solid modelling software is a significant advance in what concerns to design practise. 
The authors gratefully thank the sponsorship of Fundação para a Ciência e Tecnologia through the Strategic Project UID/EMS/00667/2013 - UNIDEMI.

\section{References}

1. G. Pahl, W. Beitz, Engineering Design: A Systematic Approach (London: The Design Council, 1988).

2. V. Hubka, Principles of engineering design, (England: Butterworth \& Co, 1982).

3. N.P. Suh, A. Bell, D. Gossard, On an axiomatic approach to manufacturing and the Design Axioms, J Eng Power-T ASME, 100: 127-130, (1978).

4. N.P. Suh, The principles of design (N.Y.: Oxford University. Press, 1990).

5. N.P. Suh, Complexity: Theory and Applications, (N.Y.: Oxford University Press, 2005).

6. A.M. Gonçalves-Coelho, G. Neştian, M. Cavique, A. Mourão, Tackling with Redundant Design Solutions Through Axiomatic Design, Int J Precis Eng Man, 13: 1837-1843, (2012).

7. C. Shannon, The mathematical theory of communication, The Bell System Technical Journal, 27: 379423, 623-656, (1948).

8. N.P. Suh, Axiomatic Design: Advances and Applications (N.Y.: Oxford University Press, 2001).

9. G.-J. Park, Analytic Methods for Design Practice (London: Springer-Verlag, 2007). 